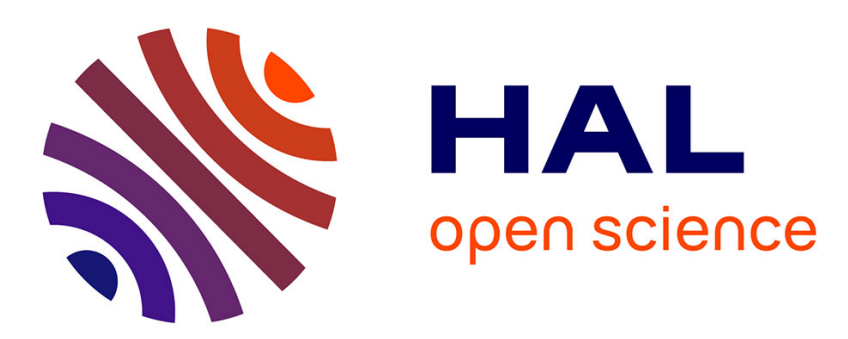

\title{
On the effect of small ions in the dynamics of polyelectrolyte solutions
}

\author{
L. Belloni, M. Drifford
}

\section{To cite this version:}

L. Belloni, M. Drifford. On the effect of small ions in the dynamics of polyelectrolyte solutions. Journal de Physique Lettres, 1985, 46 (24), pp.1183-1189. 10.1051/jphyslet:0198500460240118300 . jpa-00232955

\section{HAL Id: jpa-00232955 https://hal.science/jpa-00232955}

Submitted on 1 Jan 1985

HAL is a multi-disciplinary open access archive for the deposit and dissemination of scientific research documents, whether they are published or not. The documents may come from teaching and research institutions in France or abroad, or from public or private research centers.
L'archive ouverte pluridisciplinaire HAL, est destinée au dépôt et à la diffusion de documents scientifiques de niveau recherche, publiés ou non, émanant des établissements d'enseignement et de recherche français ou étrangers, des laboratoires publics ou privés. 
Classification

Physics Abstracts

$36.20-66.10-82.70$

\title{
On the effect of small ions in the dynamics of polyelectrolyte solutions
}

\author{
L. Belloni and M. Drifford
}

CEA-IRDI, Département de Physico-Chimie, CEN Saclay, 91191 Gif sur Yvette Cedex, France

(Reçu le 11 septembre 1985, accepté sous forme définitive le 24 octobre 1985)

\begin{abstract}
Résumé. - L'effet des petits ions sur la dynamique des solutions de polyélectrolyte est présentée dans le but de compléter une théorie parue précédemment (J. Physique Lett. 46 (1985) L-207). Plusieurs formules exprimant le coefficient de diffusion mutuelle des polyions en fonction des concentrations de polyélectrolyte et de sel ajouté sont dégagées. Pour des petits ions ponctuels, ces formules redonnent celles classiquement utilisées pour les systèmes non chargés. Un test quantitatif de ces effets est concrétisé par un bon accord avec des résultats expérimentaux obtenus en diffusion de la lumière sur des solutions de protéine.
\end{abstract}

\begin{abstract}
The effect of small ions on the dynamics of polyelectrolyte solutions complements a preceding publication (J. Physique Lett. 46 (1985) L-207). Expressions for the polyion mutual diffusion coefficient are given as a function of polyelectrolyte and added salt concentrations. For small point-like ions, the classical expressions derived for uncharged systems are deduced. A quantitative illustration of these effects is given : good agreement with light scattering measurements on protein solutions is obtained.
\end{abstract}

\section{Introduction.}

The dynamics of micellar and colloidal solutions is often studied through the so-called mutual diffusion coefficient $D_{\mathrm{m}}$, which can be measured in Quasi-elastic Light Scattering experiments (QLS). It is defined from the first cumulant of the autocorrelation function $I(q, t)$ of the scattered intensity [1] :

$$
D_{\mathrm{m}}=-\lim _{q \rightarrow 0} 1 / 2 q^{2} \frac{\partial}{\partial t} \log I(q, t=0)
$$

$\mathbf{q}$ is the diffusion vector. The limit $t=0$ in equation (1) means that the derivative is taken at times $t$ which are long compared to the time between the collisions of the solvent molecules with the particles but short compared to the characteristic times of the Brownian particle motion.

The dynamic behaviour of such solutions is affected by the thermodynamic and hydrodynamic interactions involving the continuous solvent and the polyion and small ion particles.

Many authors in the literature consider these solutions as one-component systems. In this approximation, the small ions are assumed to be point-like and to have an instantaneous diffu- 
sion constant. The theoretical treatment of uncharged hard-sphere systems is easily extended to such suspensions and the mutual diffusion constant $D_{\mathrm{m}}$ is expressed in terms of the effective polyion-polyion pair potential, like the DLVO potential [2], through classical relations [3].

On the other hand, the study of polyelectrolyte solutions as multi-component systems is much more complicated. A particular feature of charged suspensions is that one of the characteristic times is a very short relaxation time, known as the plasmon or Debye time $\tau_{\mathrm{D}}$. This time is much smaller than the experimental time in QLS. Therefore, the experimental value of $D_{\mathrm{m}}$ does not correspond to the exact definition (Eq. (1)) but to the following definition,

$$
D_{\mathrm{m}}=-\lim _{q \rightarrow 0} \frac{1}{2} q^{2} \frac{\partial}{\partial t} \log I(q, \tau)
$$

where the experimental time $\tau$ is much larger than the Debye time but remains smaller than the diffusional times of the solution. In a recent publication [4], a theoretical treatment of this pseudo mutual diffusion coefficient has been obtained by using the normal mode theory. It was shown that, in the limit of point-like ions, the classical expression for $D_{m}$ is obtained.

The purpose of this letter consists in deducing from the preceding paper, simpler and more useful expressions for $D_{\mathrm{m}}$ which account for the effect of the non-instantaneous diffusion of small ions on the polyion diffusion. Quantitative illustrations of this effect are given, first, in excess-salt solutions where a virial-type development is available, second, in low concentrated protein solutions. In the latter case, it is shown that the fit of experimental data obtained by QLS is improved in a spectacular way by introducing the finite size of the small ions.

\section{Theory.}

A polyelectrolyte solution with added salt is considered as a three-component system (polyion 1, counterion 2 and coion 3) of charged hard-spheres. The normal mode theory leads to three dynamical modes; the first one $\Gamma_{1}=\tau_{\mathrm{D}}^{-1}$ is the very fast Debye mode, the last two $\Gamma_{2}$ and $\Gamma_{3}$ are diffusional modes, proportional to $q^{2}$. For the experimental time-scale considered here, only the diffusional modes are detected, $\Gamma_{1}^{-1} \ll \tau \ll \Gamma_{2}^{-1}, \Gamma_{3}^{-1}$. The main result of the previous publication [4] gives the relation for the pseudo mutual diffusion coefficient (Eq. (22) of Ref. [4]) :

$$
D_{\mathrm{m}}=M_{11} / S_{11} \text {. }
$$

It is assumed that light is essentially scattered by the large and heavy polyions. $S_{11}=S_{11}(0)$ represents the infinite wavelength limit of the polyion-polyion structure factor $S_{11}(q)$. It is related to the pair distribution function $g_{11}(r)$ by :

$$
S_{11}=1+\rho_{1} \int\left[g_{11}(r)-1\right] \mathrm{d} \mathbf{r}
$$

$\rho_{i}$ is the number concentration of species $i(i=1,2,3)$.

The matrix $\mathbf{M}$, whose $(i, i)$ element appears in equation (2), is [4]

$$
\mathbf{M}=\mathbf{H}-1 / \Gamma_{1} \mathbf{H} \mathbf{Q}_{\mathrm{DH}} \mathbf{H} \text {. }
$$

The element $H_{i j}$ of the matrix $\mathbf{H}$ represents the hydrodynamic interactions between particles $i$ and $j$ at $q=0$. It differs from the ideal value $D_{i} \delta_{i j}$ where $D_{i}=k T / 6 \pi \eta a_{i}$ is the free-diffusion constant of the species $i$ (sphere of radius $a_{i}$ ). The hydrodynamic term $H_{j}$ cannot be expressed as simple integrals of the pair distribution functions $g_{i j}$, except for dilute solutions where the 
pair hydrodynamic interactions dominate. In this case,

$$
H_{i j}=\left[D_{i}+k T \sum_{k} \rho_{k} \int \hat{\mathbf{q}} \cdot \mu_{i k i} \cdot \hat{\mathbf{q}} g_{i \mathbf{k}} \mathrm{d} \mathbf{r}\right] \delta_{i j}+k T\left(\rho_{i} \rho_{j}\right)^{1 / 2} \int \hat{\mathbf{q}} \cdot \boldsymbol{\mu}_{i j} \cdot \hat{\mathbf{q}} g_{i j} \mathrm{~d} \mathbf{r}
$$

$\hat{\mathbf{q}}$ represents the unity vector parallel to q. $\boldsymbol{\mu}_{i k i}$ and $\boldsymbol{\mu}_{i j}$ are, respectively, the self and cross pair mobility tensors. Their explicit expression is given by Felderhof [5]. At the Oseen level, $\mu_{i k i}=0$ and $\boldsymbol{\mu}_{i j}=\mathbf{0}$ where $\mathbf{0}=1 / 8 \pi \eta r(1+\hat{\mathbf{r}} \cdot \hat{\mathbf{r}})$ is the Oseen tensor (Eq. (11) of Ref. [4]). The " DebyeHückel " matrix $Q_{\mathrm{DH}}$ is constituted by the coulombic factors $q_{i j}=4 \pi L_{\mathrm{B}} Z_{i} Z_{j}\left(\rho_{i} \rho_{j}\right)^{1 / 2} . L_{\mathrm{B}}=$ $e^{2} /\left(4 \pi \varepsilon_{0} \varepsilon k T\right)$ is the Bjerrum length, $Z_{i}$ is the charge of species $i$. The Debye mode $\Gamma_{1}$ is the trace of the matrix $\mathbf{H Q} \mathbf{Q}_{\mathrm{DH}}$.

It is important to note that the normal mode theory does not take the so-called memory or relaxation effects into account. These effects are exactly zero for the time-scale $\tau \ll \tau_{\mathrm{D}}$ but have to be explicited for $\tau_{\mathrm{D}} \ll \tau \ll \Gamma_{2}^{-1}, \Gamma_{3}^{-1}$. We think that the electrofriction described by Schurr [6] is such an effect.

What is the physical meaning of equations (2), (4) ? The first part $H_{11}$ of the term $M_{11}$ gives nothing else than the classical relation for the mutual diffusion constant $D_{\mathrm{m}}=H_{11} / S_{11}$ [1]. The second part shows the influence of small ions on the polyion motion. The diffusions of the different ions are strongly coupled since, at this time-scale $\tau \gg \tau_{\mathrm{D}}$, the local electroneutrality condition must be fulfilled. The contribution of this last part is negative which means that the polyion motion is slowed down by the non-instantaneous ionic diffusion. It disappears in the limit of point-like ions.

If the hydrodynamic interactions are introduced only through the polyion-polyion term, the expression of $D_{\mathrm{m}}$ becomes simpler :

$$
M_{11}=D_{\mathrm{m}} S_{11}=H_{11}\left[1-H_{11} q_{11} /\left(H_{11} q_{11}+D_{2} q_{22}+D_{3} q_{33}\right)\right] .
$$

Quantitative illustrations of the small ions effect are now presented.

\section{Results.}

3.1 Virial development. - In the infinite dilution limit, $\rho_{1} \rightarrow 0$, the static and dynamic properties of the solution vary linearly with the polyelectrolyte concentration. The limit $\rho_{1} \rightarrow 0$ means that the volume fraction is much smaller than unity and that the counterion concentration of the polyelectrolyte $Z_{1} \rho_{1}$ is much smaller than the added salt concentration $\rho_{\mathrm{s}}$ (excess salt limit). Different virial coefficients can be defined as :

$$
\begin{aligned}
S_{11}(0) & \sim 1+k_{\mathrm{S}} \rho_{1} \\
H_{11} & \sim D_{1}\left(1+k_{\mathrm{H}} \rho_{1}\right) \\
D_{\mathrm{m}} & \sim D_{1}\left(1+k_{\mathrm{D}} \rho_{1}\right) .
\end{aligned}
$$

The slopes $k_{\mathrm{S}}$ and $k_{\mathrm{H}}$ are given by classical integrals of the infinite dilution value $g_{11}^{0}(r)$ of the pair distribution function $g_{11}(r)$ [3]. A precise study of equation (5) shows that, even at zero polyelectrolyte concentration, the diffusion coefficient $D_{1}$ must be corrected by the self hydrodynamic factor polyion-ion-polyion. In fact, this effect is proportional to the volume fraction of the small ions and hence can be neglected. The problem is to express the $k_{\mathrm{D}}$ slope as a function of the other coefficients.

If the hydrodynamic interactions due to the small ions are neglected, equation (6) can be used and we find a first expression for $k_{\mathrm{D}}$ :

$$
k_{\mathrm{D} 1}=k_{\mathrm{H}}-k_{\mathrm{S}}-\left(Z_{1}^{2} / 2 \rho_{\mathrm{s}}\right)\left(D_{1} / D_{\mathrm{s}}\right)
$$


where $D_{4}=\left(D_{2}+D_{3}\right) / 2$. For the sake of simplicity, the ions are supposed to be monovalent. The effect of the finite ionic diffusion is clearly shown in equation (8). If this term is suppressed $\left(D_{\mathrm{s}} \rightarrow \infty\right)$, one obtains the relation for $k_{\mathrm{D}}$ which is classically used for the one-component system [3] :

$$
k_{\mathrm{DO}}=k_{\mathrm{H}}-k_{\mathrm{S}} \text {. }
$$

The last term of equation (8) does not correspond to the complete first order in $D_{1} / D_{s}$ or $a_{\mathrm{s}} / a_{1}$ of the dependence of $D_{\mathrm{m}}$ on the ionic diffusion. Indeed, the polyion-ion hydrodynamic interactions $H_{12}$ and $H_{13}$, which do not vanish for zero ion radius $a_{\mathrm{s}}$, are missing in equation (8). The exact first order in $D_{1} / D_{\mathrm{s}}$ is obtained by calculating the polyion-ion hydrodynamic factors at the Oseen level. Using this approximation, the initial slope $k_{\mathrm{D}}$ of $D_{\mathrm{m}}$ versus $\rho_{1}$ becomes, after some simple algebra :

$$
k_{\mathrm{D} 2}=k_{\mathrm{H}}-k_{\mathrm{S}}-\left[D_{1} Z_{1}-k T \rho_{\mathrm{s}} \int \hat{\mathbf{q}} \cdot \mathbf{0} \cdot \hat{\mathbf{q}}\left(g_{12}^{0}-g_{13}^{0}\right) \mathrm{d} \mathbf{r}\right]^{2} /\left(2 \rho_{\mathrm{s}} D_{\mathrm{s}} D_{1}\right)
$$

$g_{12}^{0}$ and $g_{13}^{0}$ are the values of the functions $g_{12}$ and $g_{13}$, respectively, in the limit $\rho_{1} \rightarrow 0$. It seems that the calculation of integral $I$ in equation (10) requires the knowledge of both $g_{12}^{0}$ and $g_{13}^{0}$. In fact, the integral can be performed by noting that the difference $g_{12}^{0}-g_{13}^{0}$ arises in the Poisson equation which governs the electrical potential $\psi$ around one polyion $(\varphi=e \psi / k T)$ :

$$
\begin{aligned}
\Delta \varphi=\frac{1}{r^{2}} \frac{\mathrm{d}}{\mathrm{d} r}\left(r^{2} \frac{\mathrm{d} \varphi}{\mathrm{d} r}\right) & =0 & & r<a_{1} \\
& =4 \pi L_{\mathrm{B}} \rho_{\mathrm{s}}\left(g_{12}^{0}-g_{13}^{0}\right) & & r>a_{1} .
\end{aligned}
$$

An integration by part yields for integral $I$ :

$$
I=-1 /\left(6 \pi \eta L_{\mathrm{B}} \rho_{\mathrm{s}}\right)\left[\varphi\left(a_{1}\right)+a_{1} \frac{\mathrm{d} \varphi}{\mathrm{d} r}\left(a_{1}\right)\right] .
$$

From the Gauss theorem one derives : $\mathrm{d} \varphi\left(a_{1}\right) / \mathrm{d} r=-Z_{1} L_{\mathrm{B}} / a_{1}^{2}$. We just need the surface potential $\varphi\left(a_{1}\right)$. A correct value is obtained by the linearized Poisson-Boltzmann model, $\varphi\left(a_{1}\right)=$ $Z_{1} L_{\mathrm{B}} / a_{1} /\left(1+\kappa a_{1}\right)$. With this value, the slope $k_{\mathrm{D}}$ becomes

$$
k_{\mathrm{D} 2}=k_{\mathrm{H}}-k_{\mathrm{S}}-\left(Z_{1}^{2} / 2 \rho_{\mathrm{s}}\right)\left(D_{1} / D_{\mathrm{s}}\right) /\left(1+\kappa a_{1}\right)^{2}
$$

$\kappa=\left(8 \pi L_{\mathrm{B}} \rho_{\mathrm{s}}\right)^{1 / 2}$ is the Debye screening parameter. Equation (13) differs from equation (8) by the $\left(1+\kappa a_{1}\right)^{2}$ factor.

The last development is similar to the calculation of the electrophoretic effect in simple electrolytes.

In order to estimate the magnitude of the last term in equations (8) and (13) relative to the classical term $k_{\mathrm{H}}-k_{\mathrm{S}}$, the slopes $k_{\mathrm{H}}$ and $k_{\mathrm{S}}$ must be calculated. This requires simple numerical integrals of the polyion-polyion distribution function $g_{11}^{0}(r)=\exp \left[-\beta v_{11}^{\text {eff }}(r)\right]$, where $v_{11}^{\text {eff }}(r)$ is the effective pair potential. Numerous expressions of this potential are presented in the literature. A correct and very simple form is [2] :

$$
\begin{aligned}
\beta v_{11}^{\text {eff }}(r) & =+\infty & & r<2 a_{1} \\
& =Z_{1}^{2} L_{\mathrm{B}} /\left(1+\kappa a_{1}\right)^{2} \exp \left[-\kappa\left(r-2 a_{1}\right)\right] / r & & r>2 a_{1} .
\end{aligned}
$$

In the Debye-Hückel limit (point charge $a_{1}=0$ and linearization of the exponential exp $(-\beta v)$ ), the analytical result is, $k_{\mathrm{H}}=0, k_{\mathrm{s}}=-Z_{1}^{2} / 2 \rho_{\mathrm{s}}$ and

$$
k_{\mathrm{D} 3}=Z_{1}^{2} / 2 \rho_{\mathrm{s}}\left(1-D_{1} / D_{\mathrm{s}}\right) \text {. }
$$


The relative magnitude of the $D_{1} / D_{\mathrm{s}}$ term is clear here. This simple expression for the slope $k_{\mathrm{D}}$, which has been used by many authors [7], is correct for simple electrolytes but fails for polyelectrolytes. The linearization in the Debye-Hückel approximation leads to an overestimate in the value of $k_{\mathrm{H}}-k_{\mathrm{S}}$. Thus, the fit of experimental data with equation (15) always gives values of the polyion charge which are too low.

The differences between the calculated values of $k_{\mathrm{D} 0}, k_{\mathrm{D} 1}$ and $k_{\mathrm{D} 2}$ are illustrated in table $\mathrm{I}$. The physical parameters are $L_{\mathrm{B}}=7.19 \AA(T=298 \mathrm{~K}), a_{1}=25 \AA\left(D_{1} \sim 10^{-6} \mathrm{~cm}^{2} / \mathrm{s}\right)$ and $Z_{1}=30$, which is a correct representation of many aqueous micellar solutions. The values of $k_{\mathrm{D}}$ are given for different salt concentrations, $0.1 \mathrm{M}, 0.01 \mathrm{M}$ and $0.001 \mathrm{M}$.

Expression (15) clearly fails for these highly charged systems. The linearization in the DebyeHückel treatment is incorrect because of the strong electrostatic repulsion between polyions. The fit of the values $k_{\mathrm{Do}}$ with equation (15) is obtained with charges which are listed in table I. These charges are about half the real charge.

The comparison of the slopes $k_{\mathrm{D} 1}$ and $k_{\mathrm{D} 2}\left(D_{1} / D_{\mathrm{s}}=a_{\mathrm{s}} / a_{1}=1 / 10\right)$ with the classical expression $k_{\mathrm{D} 0}$ leads to several conclusions :

First, the $k_{\mathrm{D} 1}$ and $k_{\mathrm{D} 2}$ slopes are different, especially at large salinity, arising from the $\left(1+\kappa a_{1}\right)^{2}$ factor in equation (13). The suppression of the polyion-ion hydrodynamic interactions overestimates the effect of ionic diffusion. In some cases, the correction $Z_{1}^{2} / 2 \rho_{\mathrm{s}} D_{1} / D_{\mathrm{s}}$ is larger than $k_{\mathrm{D} 0}$ and the slope $k_{\mathrm{D} 1}$ becomes negative thus giving false results. The correct expression is then equation (13).

The difference of $k_{\mathrm{D} 2}$ with $k_{\mathrm{D} 0}$ becomes appreciable with decreasing salt concentration. The relative magnitude of the correction in $D_{1} / D_{\mathrm{s}}$ compared to $k_{\mathrm{H}}-k_{\mathrm{S}}$ is $23 \%$ at $10^{-3} \mathrm{M}$ salinity although $D_{1} / D_{\mathrm{s}}=10 \%$. It increases with increasing polyion charge and decreases with increasing size.

This effect is illustrated by experimental results in micellar solutions with low added salt concentration [8] : experimental data of $k_{\mathrm{D}}$ are always smaller than the theoretical $k_{\mathrm{D} 0}$ but are well fitted with expression (13).

3.2 LOW CONCENTRATED PROTEIN SOLUTIONS. - An interesting test of the present theory is realized by examining the results of Neal, Purich and Cannell [9]. These authors have measured, with fine light scattering experiments, the static structure factor $S_{11}$ and the mutual diffusion coefficient $D_{\mathrm{m}}$ for solutions of charged bovine serum albumin at different macromolecule charges and ionic strengths. The solutions are dilute with regard to the volume fraction $(\sim 0.5 \%$ with $a_{1}=35.3 \AA$ ), but not with regard to the electrostatic interactions since the ionic strength is very low. In some cases, the counterions which equilibrate the charge of the polyions are far more numerous than those which come from the added salt. These solutions cannot be considered as excess salt solutions and the linear expressions (7) are not valid : the theoretical values

Table I. - Theoretical values of the slopes $k_{\mathrm{S}}, k_{\mathrm{H}}$ and $k_{\mathrm{D}}$ (see Eq. (7)), expressed in $\mathrm{mol}^{-1} 1$, at different added salt concentrations. $a_{1}=25 \AA, Z_{1}=30$ and $D_{1} / D_{\mathrm{s}}=1 / 10 . k_{\mathrm{D} 0}=k_{\mathrm{H}}-k_{\mathrm{s}} \cdot k_{\mathrm{D} 1}$ and $k_{\mathrm{D} 2}$ are given by equations (8) and (13). The values $Z_{\mathrm{fit}}$ represent the erroneous charges which fit the values of $k_{\mathrm{D} 0}$ with the expression $Z_{1}^{2} / 2 \rho_{\mathrm{s}}$.

\begin{tabular}{lrrrrrrrr}
\hline \multicolumn{2}{c}{$\rho_{\mathrm{s}}$} & $-k_{\mathrm{S}}$ & $-k_{\mathrm{H}}$ & \multicolumn{1}{c}{$k_{\mathrm{D} 0}$} & $Z_{1}^{2} / 2 \rho_{\mathrm{s}}$ & $k_{\mathrm{D} 1}$ & $k_{\mathrm{D} 2}$ & $Z_{\mathrm{fit}}$ \\
\hline 0.1 & $\mathrm{M}$ & 1090 & 535 & 555 & 4500 & 105 & 520 & 10.5 \\
0.01 & $\mathrm{M}$ & 9230 & 2120 & 7110 & 45000 & 2610 & 5760 & 11.9 \\
$0.001 \mathrm{M}$ & 132200 & 12200 & 120000 & 450000 & 75000 & 91700 & 15.5 \\
\hline
\end{tabular}


of $S=S_{11}$ and $H_{11}$ must be calculated from the distribution functions (Eqs. (3) and (5)), computed with the integral equations HNC [10] or RMSA [11].

Neal et al. have found a good adjustment for the structure factor data but a significant deviation for the diffusion coefficient data, especially at low ionic strength. This discrepancy is shown in figure 1 where their measured and calculated values of $S D_{\mathrm{m}} / D^{0}$ are plotied versus $S^{-1}$ (Fig. 8 of Ref. [9]). Their theoretical curve corresponds to $S D_{\mathrm{m}} / D^{0}=H_{11} / D^{0}$. Since the voiume fraction is negligible, the misfit does not result from the fact that the hydrodynamic interactions are included to first order in the concentration. Agreement is obtained by taking account of the non-instantaneous diffusion of ions, namely by using equation (6) (in such solutions, the polyionion hydrodynamic interactions can be neglected since $\left.\kappa a_{1} \ll 1\right)$. With $D_{2}=D_{3}=D_{s}$, the correct expression is then

$$
S D_{\mathrm{m}} / D^{0}=H_{11} / D^{0}\left[1-H_{11} Z_{1} \rho_{1} /\left(H_{11} Z_{1} \rho_{1}+2 I D_{\mathrm{s}}\right)\right]
$$

$I=\rho_{\mathrm{s}}+Z_{1} \rho_{1} / 2$ is the ionic strength.

The theoretical curve given by equation (16) can be obtained, in a very simple manner, from the original curve of Neal et al. This curve is plotted on the same figure 1, for two values of the ionic radius $a_{\mathrm{s}}, 1.5 \AA$ and $2 \AA$. As was noted by Neal et al. [9], different solutions which lead to identical values of $S$ also lead to similar values of $D_{\mathrm{m}}$. It is clear that the theoretical diffusion coefficients are reduced, due to the nonzero size of small ions, and tend to the experimental data. The best agreement is obtained with $a_{\mathrm{s}}=1.5 \AA$, which is a reasonable value $\left(D_{\mathrm{s}} \sim 14 \times 10^{-6} \mathrm{~cm}^{2} / \mathrm{s}\right.$ $\left.\sim D_{\mathrm{Na}^{+}}\right)$.

In the absence of salt (the linear coefficients $k_{\mathrm{S}}$ and $k_{\mathrm{H}}$ diverge), the interactions through $S^{-1}$ are maximum and the mutual diffusion coefficient tends to the Nernst-Hartley value, $S D_{\mathrm{m}}=$ $H_{11} D_{2} /\left(H_{11} Z_{1}+D_{2}\right)$.

\section{Conclusion.}

Simple expressions for the ionic diffusion effect in the dynamics of polyelectrolyte solutions have been given. The relative magnitude of this correction was shown to be appreciable at low salinity.

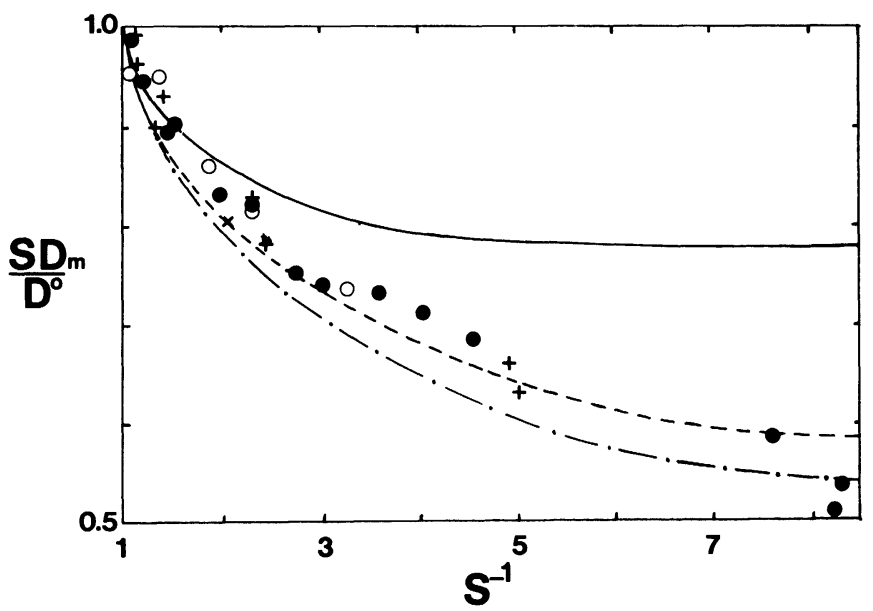

Fig. 1. - Experimental and theoretical values for the quantity $S D_{\mathrm{m}} / D^{0} v s . S^{-1} . S$ represents the long wavelength limit of the polyion-polyion structure factor, $S_{11}(0)$. $D_{\mathrm{m}}$ is the mutual diffusion coefficient, $D^{0}$ its value in absence of interaction. The symbols represent the experimental data of Ref. [9]. The classical theory is shown as a solid line. The noninstantaneous diffusion of small ions is introduced via equation (16), with an ionic radius $a_{\mathrm{s}}=1.5 \AA(--)$ and $a_{\mathrm{s}}=2 \AA(-.-)$. 
A good improvement for the fit of experimental results has been obtained. In view to get a more precise picture of the dynamics of charged suspensions, the relaxation of the small ions around the polyions will have to be introduced.

\section{References}

[1] Ackerson, B. J., J. Chem. Phys. 64 (1976) 242.

[2] VERWEY, E. J. W. and OVERBEEK, J. T. G., Theory of the stability of lyophobic colloids (Elsevier, Amsterdam) 1948.

[3] Corti, M. and Degiorgio, V., J. Phys. Chem. 85 (1981) 711.

[4] Belloni, L., Drifford, M. and TurQ, P., J. Physique Lett. 46 (1985) L-207.

[5] Felderhof, B. U., Physica A 89 (1977) 373.

[6] Schurr, J. M., Chem. Phys. 45 (1980) 119.

[7] LeE, W. I. and Schurr, J. M., J. Polym. Sci. 13 (1975) 873.

[8] Drifford, M., Belloni, L., Dalbiez, J. P. and Chattopadhyay, A., J. Colloid Interface Sci. 105(1985) 587.

[9] Neal, D. G., Purich, D. and Cannell, D. S., J. Chem. Phys. 80 (1984) 3469.

[10] Belloni, L., Chem. Phys. 99 (1985) 43.

[11] Hayter, J. B. and Penfold, J., Mol. Phys. 42 (1981) 109. 